\title{
Acute renal failure due to sulphadiazine crystalluria in AIDS patients
}

\author{
P de Sequera, M Albalate, J Hernandez, A Vazquez, J Abad, E Ramiro, M Fernández \\ Guerrero, C Caramelo, $S$ Casado, A Ortiz
}

\begin{abstract}
Summary
We report two AIDS patients who developed acute renal failure while receiving sulphadiazine for cerebral toxoplasmosis. Renal ultrasound revealed diffuse bilateral echogenic shadowing material. 'Sheaves of wheat' crystals, typical of sulphadiazine crystalluria, were present in the urine. One patient required a percutaneous nephrostomy. Hydration and urine alkalinisation resulted in rapid improvement of renal function and ultrasonographic findings. Sulphadiazine-induced crystalluria and acute renal failure is increasingly frequent. Awareness of its existence may lead to prevention and early conservative treatment.
\end{abstract}

Keywords: sulphadiazine, crystalluria, renal failure, AIDS

Sulphonamides are among the drugs that may precipitate in acid urine and give rise to crystalluria and calculi. In recent years, sulphadiazine, in association with pyrimethamine, has become standard therapy for cerebral toxoplasmosis, a frequent complication of the acquired immune deficiency syndrome (AIDS) and other immunodeficiency states. ${ }^{1}$ The progression of the AIDS epidemic has been accompanied by an increased use of sulphadiazine, and a re-emergence of its adverse effects. Renal failure is one of these 'forgotten' complications. $^{2}$

Fundación Jiménez Díaz, Madrid, Spain Nephrology Service $\mathrm{P}$ de Sequera $M$ Albalate

J Hernandez

A Vazquez

C Caramelo

S Casado

A Ortiz

Radiology Department J Abad

E Ramiro

Infectious Diseases

Unit

M Fernández Guerrero

Correspondence to

Alberto Ortiz, S Nefrología, Fundación Jiménez Díaz,

Av Reyes Católicos 2, 28040 Madrid, Spain

Accepted 22 November 1995
Case 1

An HIV-positive 31-year-old man presented with vomiting, left flank pain that radiated to the groin, oligoanuria and dark urine. An evaluation one month before admission for fever and right hemiparesis disclosed an intracerebral mass suggestive of cerebral toxoplasmosis. Sulphadiazine $(1 \mathrm{~g} / 6 \mathrm{~h})$, pyrimethamine (25 mg/day), and folinic acid were begun seven days before admission. At that time renal function was normal (serum creatinine $88 \mu \mathrm{mol} / \mathrm{l}$ ).

On admission, his body temperature was $36^{\circ} \mathrm{C}$ and blood pressure $120 / 80 \mathrm{mmHg}$. Significant initial laboratory values were: creatinine $858 \mu \mathrm{mol} / 1$, urea $22 \mu \mathrm{mol} / 1$. Urinalysis revealed gross haematuria, and many crystals with a characteristic 'sheaves of wheat' morphology (figure 1). A plain abdominal X-ray showed normal-sized kidneys with no evidence of calculi. Renal ultrasound demonstrated bilateral multiple diagnostic echogenic foci with shadowing consistent with calculi. There was no hydronephrosis. Diuresis for the first 24 hours was $200 \mathrm{ml}$.

Sulphadiazine was discontinued and he was intravenously hydrated with $1 / 6 \mathrm{M} \mathrm{NaHCO}_{3}$ and $0.9 \%$ sodium chloride. After $24 \mathrm{~h}$ he was challenged with $250 \mathrm{mg}$ furosemide intravenously, and diuresis over the next 24 hours was 61 . Urine $\mathrm{pH}$ was 7.5. Renal function improved rapidly over the next several days. On day 8 , urea was $3.8 \mu \mathrm{mol} / 1$; creatinine $92 \mu \mathrm{mol} /$ 1 , and a repeat ultrasound revealed absence of calculi.

Case 2

A 57-year-old man presented with abdominal pain, dysuria and oligoanuria. One month before admission he had been diagnosed as having AIDS and cerebral toxoplasmosis, and had been discharged three days earlier on sulphadiazine $(1.5 \mathrm{~g} / 6 \mathrm{~h})$, pyrimethamine (25 mg/day), and folinic acid.

On admission, his body temperature was $37.3^{\circ} \mathrm{C}$ and blood pressure $140 / 80 \mathrm{mmHg}$. Initial laboratory values were: creatinine $619 \mu \mathrm{mol} / 1$, urea $21 \mu \mathrm{mol} / 1$. Urinalysis revealed haematuria and 'sheaves of wheat' crystals. A simple abdominal X-ray showed normal-sized kidneys with no evidence of calculi. Renal ultrasound demonstrated bilateral multiple echogenic foci with shadowing consistent with calculi, and minimal bilateral dilatation of the renal pelvis (figure 2). Sulphadiazine was discontinued. Intravenous hydration with 1/ $6 \mathrm{M} \mathrm{NaHCO}_{3}$ and $0.9 \%$ sodium chloride was begun, but the patient remained anuric for the

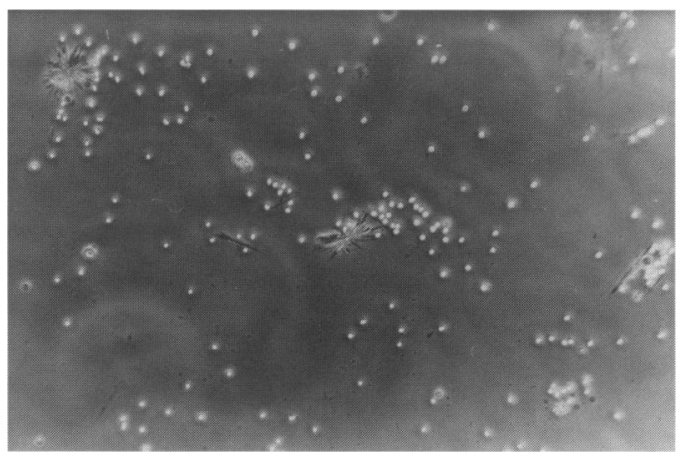

Figure 1 Characteristic 'sheaves of wheat' crystals and numerous red blood cells in urine 
Figure 2 Right kidney sonogram on admission: hyperechogenic images of $7.5 \mathrm{~mm}$ (arrow) with acoustic shadowing and moderate hydronephrosis (asterisks)

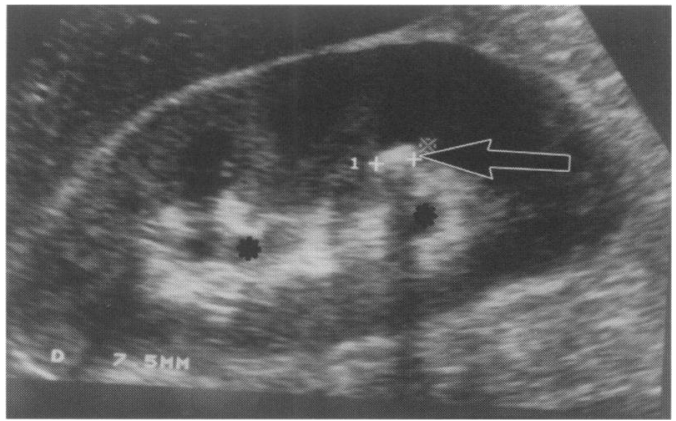

\section{Sulphadiazine crystalluria}

- aetiology: therapeutic sulphadiazine doses

- manifestations: renal colic and acute renal failure

- prevention: adequate hydration and urine alkalinisation

- diagnosis: renal sonogram and characteristic urinary crystals

- treatment: hydration and urine alkalinisation

first 12 hours. A left nephrostomy catheter was inserted and minimal diuresis was obtained. Brisk diuresis was only observed after an intravenous furosemide $250 \mathrm{mg}$ challenge. Thereafter, renal function and urinary output improved rapidly. On day 6 , creatinine was $132 \mu \mathrm{mol} / 1$, and a repeat ultrasound revealed absence of calculi.

\section{Discussion}

Obstructive renal failure associated with sulphadiazine has been recognised since the 1940 s. $^{3,4}$ However, with the advent of newer, safer antibiotics, this complication all but disappeared from the medical literature, only to re-emerge in the wake of the AIDS epidemic. ${ }^{1,5,6}$ Sulphonamides precipitate in the urinary tract due to their low solubility in acid media, especially in the presence of dehydration and hypoalbuminaemia, ${ }^{6}$ two conditions that are common in AIDS patients. Sulphadiazine has a relatively low urine solubility, so that for the usual daily dose of $4 \mathrm{~g}$, approximately 161 of urine at a pH of 5.5 need to be produced to keep the drug in solution. ${ }^{2}$ The amount of sulphadiazine excreted without crystallisation can be increased more than 20fold by increasing urinary $\mathrm{pH}$ from 5.5 to $7.5 .^{3}$

Renal toxicity usually develops after three days of sulphadiazine therapy, although it has been reported following a single large dose. ${ }^{2}$ Sulphadiazine crystalluria should be suspected when acute renal failure develops in patients receiving sulphadiazine, especially if there are symptoms of renal colic. Confirmation of the diagnosis requires demonstration of radiolucent urinary lithiasis by ultrasound and plain abdominal $\mathrm{X}$-ray, ${ }^{7}$ as well as urinalysis that

1 Simon DL, Brosius BC, Rothstein DM. Sulfadiazine crystalluria revisited. Arch Intern Med 1990; 150: 2379-84. 2 Dorfman L, Smith J. Sulfonamide crystalluria: a forgotten disease. F Urol 1970; 104: 482-3.

3 Abeshouse BS, Tankin LH. Renal complications of sulfonamide therapy. F Urol 1946; 56: 658-87.

4 Finland M, Strauss E, Peterson EL. Sulfadiazine. Therapeutic evaluation and toxic effects on four hundred and apeutic evaluation and toxic effects on four hun

5 Rivera $M$, Liaño $\mathrm{F}$, Fortún J, Fernández-Lucas $\mathrm{M}$, Ortuño

5 Rivera M, Liaño F, Fortún J, Fernández-Lucas M, Ortuño
J. Sulfadiazine-induced obstructive renal failure in a patient with acquired immunodeficiency syndrome (AIDS). Nephrol Dial Transplant 1993; 8: 1183-4.
Renal hypoperfusion

- vomiting, diarrhoea

Intrinsic renal failure

- renal infection and sepsis

- nephrotoxicity: aminoglycosides, amphotericin B, pentamidine, radiocontrast agents

- allergic interstitial nephritis: trimethoprim sulphametaxazole

- renal infiltration by neoplasm

- HIV-associated nephropathy and other glomerulonephritis

Obstructive uropathy/intratubular deposition of crystals

- sulphadiazine, acyclovir

reveals the characteristic 'sheaves of wheat' crystals. ${ }^{1,4}$ A high degree of clinical suspicion and early diagnosis may lead to prompt conservative treatment with alkaline hydration and may prevent the need for renal biopsy, dialysis or invasive urologic manipulation, all of which have been employed in reported cases of sulphadiazine crystalluria. Alkaline diuresis should always be induced, as intratubular sulphadiazine deposition ${ }^{8}$ will not be relieved by urinary catheterisation. However, in severe cases, urinary catheterisation and/or lavage with $5 \%$ sodium bicarbonate may be required. ${ }^{2}$

In conclusion, clinicians caring for AIDS patients should be aware of the potential of sulphadiazine to cause acute renal failure. Prophylactic adequate hydration and urine alkalinisation should be ensured in patients receiving sulphadiazine.

6 Sahai J, Heimberger T, Collins K, Kaplowitz L, Polk R. Sulfadiazine-induced crystalluria in a patient with the acquired immunodeficiency syndrome: a reminder. $A m \mathcal{F}$ Med 1988; 84: $791-2$.

7 Sasson JP, Dratch PL, Shortsleeve MJ. Renal US findings in sulfadiazine-induced crystalluria. Radiology 1992; 185: $739-40$.

8 Portoles J, Torralbo A, Prats D, Blanco J, Barrientos A. Acute renal failure and sulphadiazine crystalluria in kidney transplant. Nephrol Dial Transplant 1994; 9: 180-1.

\section{Renal failure in HIVIAIDS}

